\title{
Quantum properties of exponential states
}

\author{
Alfredo Luis* \\ Departamento de Óptica, Facultad de Ciencias Físicas, Universidad Complutense, 28040 Madrid, Spain
}

(Received 31 March 2007; published 30 May 2007)

\begin{abstract}
The use of Rényi entropy as an uncertainty measure alternative to variance leads to the study of states with quantum fluctuations below the levels established by Gaussian states, which are the position-momentum minimum uncertainty states according to variance. We examine the quantum properties of states with exponential wave functions, which combine reduced fluctuations with practical feasibility.
\end{abstract}

DOI: 10.1103/PhysRevA.75.052115

\section{INTRODUCTION}

The most widely used measure of uncertainty is variance, which has good properties that include simple uncertainty relations for basic variables such as position and momentum or the quadratures of a single-mode field. The minimum uncertainty states for position and momentum have Gaussian wave functions, combining interesting theoretical properties with practical feasibility. These are coherent and squeezed states. For mechanical systems, such as a trapped ion, this includes the ground state of harmonic potentials, their displacement created by a sudden shift of the trap center, and the result of a nonadiabatic drop in the trap spring constant [1]. In quantum optics they include the vacuum state, the output of a laser well above threshold, and light emerging from nonlinear media illuminated by a laser beam [2].

However, variance is not free from nontrivial difficulties. The most relevant are that (i) it puts too much weight on the tails of probability distributions, and (ii) it leads to meaningless infinite variances in common situations [3]. Among other possibilities, these difficulties are avoided by the Rényi entropy, which has been successfully applied in recent times to diverse physical problems [4-8].

The analysis of the ultimate quantum limits using this measure of uncertainty leads to the study of states with nonGaussian wave functions. In this work we examine the quantum properties of states with exponential wave functions, which combine reduced fluctuations with practical feasibility. These states are feasible since the exponential wave functions are the ground state of $\delta$ potentials, which can be approximated in practice by square potential wells in the limit of vanishing width $L$ and large depth $V$ with finite product $V L[9]$.

More specifically, we show that, when using the Rényi entropy, the position-momentum uncertainty product for exponential states is less than for Gaussian states, which are the minimum position-momentum uncertainty states according to variance (Sec. III). We show that they are nonclassical states, as revealed by their nonpositive Wigner functions (Sec. IV), the presence of squeezing (Sec. V), and subPoissonian statistics (Sec. VI). We examine the application

*Electronic address: alluis@ fis.ucm.es; URL: http://www.ucm.es/ info/gioq of these states to the detection of small phase shifts, showing that they reach the Heisenberg limit (Sec. VII).

\section{RÉNYI ENTROPY}

We consider a one-dimensional problem describable by two conjugate Cartesian variables $X, P$ satisfying the commutation relation $[X, P]=i \hbar$. In order to cover different physical situations (such as position and momentum variables of a trapped ion or the field quadratures of a singlemode quantum light field) we will consider the dimensionless variables

$$
x=\sqrt{\frac{m \omega}{\hbar}} X, \quad p=\frac{1}{\sqrt{m \omega \hbar}} P,
$$

with $[x, p]=i$, where $m$ is a mass and $\omega$ a frequency.

The most popular measure of uncertainty of a generic observable $A$ is the variance $\Delta A$,

$$
(\Delta A)^{2}=\sum_{a}(a-\langle A\rangle)^{2} \mathcal{P}(a), \quad\langle A\rangle=\sum_{a} a \mathcal{P}(a),
$$

where $a$ are the values of the observable $A$ and $\mathcal{P}(a)$ are the corresponding probabilities. The main advantage of this measure is that it leads to an universal uncertainty relation for $x$ and $p$,

$$
\Delta x \Delta p \geqslant \frac{1}{2} .
$$

Nevertheless, the product of variances becomes trivial and useless in finite-dimensional spaces since there are proper states with $\Delta A=0$ [10].

The main drawback of the variance is the tail effect, where values of $A$ with vanishing probabilities $\mathcal{P}(a)$ but large $|a|$ are overestimated. This is particularly relevant for practical experimental and numerical problems, since the accurate determination of the tails $|a| \rightarrow \infty$ with $\mathcal{P}(a) \rightarrow 0$ requires large resources.

As a further disadvantage, the variance leads very often to meaningless diverging results $\Delta A \rightarrow \infty$. To illustrate this point let us consider a simple case where only two outcomes $a$ $=0, a_{0}$ have nonvanishing probabilities,

$$
\mathcal{P}\left(a_{0}\right)=\sigma, \quad \mathcal{P}(0)=1-\sigma,
$$

with $\mathcal{P}\left(a \neq 0, a_{0}\right)=0$, so we get 


$$
\Delta A=\langle A\rangle \sqrt{\frac{1}{\sigma}-1} .
$$

For fixed $\langle A\rangle=\sigma a_{0}$, we have $\Delta A \rightarrow \infty$ when $\sigma \rightarrow 0$ and $a_{0}$ $\rightarrow \infty$. This behavior is rather paradoxical, because the divergence of $\Delta A$ occurs in the limit in which the state becomes the eigenstate of $A$ with eigenvalue $a=0$, since when $\sigma \rightarrow 0$ we have $\mathcal{P}(0) \rightarrow 1$.

As a suitable alternative to the variance avoiding the above difficulties, we may consider, for instance, the $\eta=2$ case of the family of Rényi entropies [4]

$$
H_{\eta}=\frac{1}{1-\eta} \ln \left(\sum_{a} \mathcal{P}^{\eta}(a)\right)
$$

so that we define the uncertainty $\delta A$ of the observable $A$ as [4-8]

$$
\delta A=e^{H_{2}}=\frac{1}{\sum_{a} \mathcal{P}^{2}(a)},
$$

which is bounded from below by unity, $\delta A \geqslant 1$.

Uncertainty relations involving this uncertainty measure are available for finite-dimensional spaces of arbitrary dimension $N[6,7]$. For example, in Ref. [6] it has been shown that, for complementary observables $A$ and $B$, with $|\langle a \mid b\rangle|$ $=1 / \sqrt{N}$, where $|a\rangle$ and $|b\rangle$ are the corresponding eigenvectors, there are uncertainty relations of the form (see the Appendix for further details of this derivation)

$$
\delta A \delta B \geqslant\left(\frac{2 N}{N+1}\right)^{2} .
$$

However, to the best of our knowledge, there is no known counterpart of this uncertainty relation for observables with a continuous spectrum in infinite-dimensional spaces [7].

\section{EXPONENTIAL STATES AND UNCERTAINTY PRODUCTS}

Let us consider the family of exponential states $\psi(x)$

$$
\psi(x)=\sqrt{\frac{2^{1 / \alpha} \alpha}{2 \gamma \Gamma(1 / \alpha)}} \exp \left(i\langle p\rangle x-\frac{|x-\langle x\rangle|^{\alpha}}{\gamma^{\alpha}}\right),
$$

where $\gamma$ and $\alpha$ are real positive parameters. For arbitrary $\alpha, \gamma$ we have

$$
\Delta x=\frac{\gamma}{2^{1 / \alpha}} \sqrt{\frac{\Gamma(3 / \alpha)}{\Gamma(1 / \alpha)}}, \quad \Delta p=\frac{\alpha 2^{1 / \alpha}}{2 \gamma} \sqrt{\frac{\Gamma(2-1 / \alpha)}{\Gamma(1 / \alpha)}},
$$

and

$$
\delta x=\frac{2 \gamma}{\alpha} \Gamma(1 / \alpha)
$$

while no simple analytical expression for $\delta p$ is available, leaving aside the particular cases $\alpha=1,2, \infty$.

This family includes the Gaussian states $\psi_{g}(x)$ for $\alpha=2$,

$$
\psi_{g}(x)=\left(\frac{2}{\pi \gamma^{2}}\right)^{1 / 4} \exp \left(i\langle p\rangle x-\frac{(x-\langle x\rangle)^{2}}{\gamma^{2}}\right),
$$

which reach the equality in the uncertainty relation (2.3) with

$$
\Delta_{g} x=\frac{\gamma}{2}, \quad \Delta_{g} p=\frac{1}{\gamma}
$$

so that they are referred to as minimum uncertainty states. Concerning the Rényi entropy, we have

$$
\delta_{g} x=\gamma \sqrt{\pi}, \quad \delta_{g} p=\frac{2}{\gamma} \sqrt{\pi} .
$$

Relevant examples of Gaussian states are the coherent states $\psi_{c}(x)$, which are defined by $\alpha=2$ and $\gamma=\sqrt{2}$,

$$
\psi_{c}(x)=\frac{1}{\pi^{1 / 4}} \exp \left(i\langle p\rangle x-\frac{(x-\langle x\rangle)^{2}}{2}\right),
$$

so that $\Delta_{c} x$ and $\Delta_{c} p$ are equal,

$$
\Delta_{c} x=\Delta_{c} p=\frac{1}{\sqrt{2}},
$$

and also $\delta_{c} x$ and $\delta_{c} p$ are equal,

$$
\delta_{c} x=\delta_{c} p=\sqrt{2 \pi} .
$$

Gaussian states are no longer minimum uncertainty states when using the Rényi entropy (2.7), so that there are states with uncertainty products $\delta x \delta p$ below $2 \pi$ [8]. Maybe the simplest and most interesting case is given by the exponential states with $\alpha=1$,

$$
\psi_{e}(x)=\frac{1}{\sqrt{\gamma}} \exp \left(i\langle p\rangle x-\frac{|x-\langle x\rangle|}{\gamma}\right),
$$

or, in momentum representation,

$$
\tilde{\psi}_{e}(p)=\sqrt{\frac{2 \gamma}{\pi}} \frac{\exp (-i p\langle x\rangle)}{1+\gamma^{2}(p-\langle p\rangle)^{2}},
$$

for which

$$
\Delta_{e} x=\frac{\gamma}{\sqrt{2}}, \quad \Delta_{e} p=\frac{1}{\gamma}
$$

and

$$
\delta_{e} x=2 \gamma, \quad \delta_{e} p=\frac{4 \pi}{5 \gamma} .
$$

When using the Rényi measure (2.7) the $\alpha=1$ states $\psi_{e}(x)$ in Eq. (3.10) present a product of uncertainties below the Gaussian level,

$$
\delta_{e} x \delta_{e} p=\frac{8 \pi}{5}<\delta_{g} x \delta_{g} p=2 \pi
$$

while the opposite result is obtained for the product of variances, 


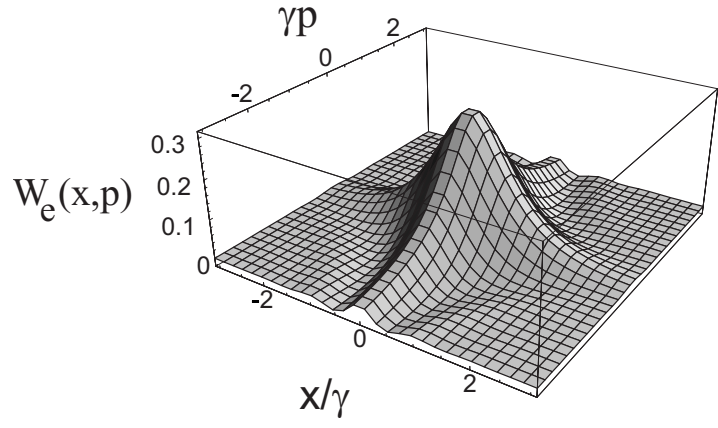

FIG. 1. Wigner function $W_{e}(x, p)$ for the exponential state (3.10) for $\langle x\rangle=\langle p\rangle=0$ as a function of $x / \gamma$ and $\gamma p$.

$$
\Delta_{e} x \Delta_{e} p=\frac{1}{\sqrt{2}}>\Delta_{g} x \Delta_{g} p=\frac{1}{2} .
$$

The reduction of fluctuations for exponential states with $\alpha$ $\neq 1$ increases when $\alpha$ decreases, as shown in Ref. [8], where these states have been studied in classical optics as waves less diffracting than Gaussian waves.

\section{NONCLASSICAL BEHAVIOR}

The amount of nonclassical behavior can be measured in different ways. One of the most widely used relies on the positivity or negativity of $s$-ordered quasidistributions on the phase space of the problem that includes the Wigner function $[11,12]$. From a practical perspective, this is reflected in phenomena such as squeezing and sub-Poissonian statistics, which are examined in more detail below.

For the exponential states (3.1) with $\alpha \neq 2$ the Wigner function takes always negative values, since the Gaussian states are the only pure states with positive definite Wigner function [13]. In particular, for the case $\alpha=1$ the Wigner function is (for $\langle x\rangle=\langle p\rangle=0$ without loss of generality)

$$
\begin{aligned}
W_{e}(x, p)= & \frac{1}{\pi} \exp \left(-2 \frac{|x|}{\gamma}\right)\left(\frac{\cos (2 p|x|)-\gamma p \sin (2 p|x|)}{1+(\gamma p)^{2}}\right. \\
& \left.+\frac{\sin (2 p|x|)}{\gamma p}\right)
\end{aligned}
$$

which is represented in Fig. 1 as a function of $x / \gamma$ and $\gamma p$. The amount of negativity can be measured, for example, in terms of the distance between $W_{e}(x, p)$ and its modulus $[12,14]$,

$$
N=\int_{-\infty}^{\infty} d p \int_{-\infty}^{\infty} d x\left[W_{e}(x, p)-\left|W_{e}(x, p)\right|\right]^{2},
$$

which in our case leads to

$$
N \simeq 2.5 \times 10^{-3} .
$$

This negativity is actually very small when compared to the negativity of number states, which is two orders of magnitude larger, even for small photon numbers such as $n \leqslant 10$ [14]. This measure of negativity is not very informative since, for example, it does not depend on the amount of

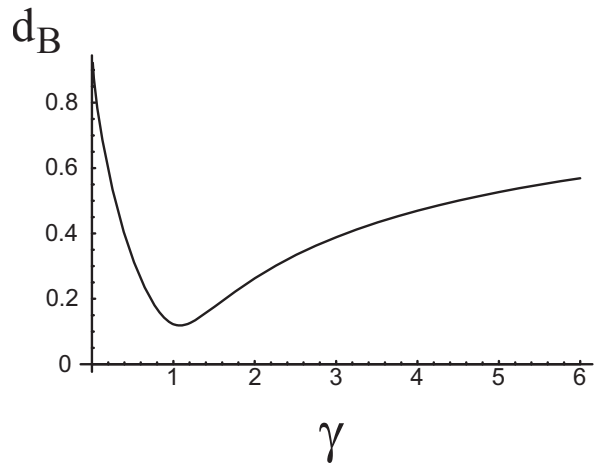

FIG. 2. $d_{B}$ as a function of $\gamma$ for the exponential state (3.10). Minimum occurs around $\gamma \simeq 1.08$.

squeezing represented by the parameter $\gamma$. A more informative measure is provided by the distance between $\psi_{e}$ and the coherent states $\psi_{c}$, which are reported to be the only classical pure states $[13,15]$. This can be carried out, for example, in terms of the Bures distance

$$
d_{B}^{2}=\frac{1}{2} \min _{c}\left\|\psi_{e}-\psi_{c}\right\|^{2}=1-\max _{c} \sqrt{F},
$$

where $F$ is the fidelity,

$$
F=\left|\int d x \psi_{c}^{*}(x) \psi_{e}(x)\right|^{2},
$$

and $\min _{c}\left(\max _{c}\right)$ means that we consider the minimum (maximum) by varying the state parameters $\langle x\rangle_{c}$ and $\langle p\rangle_{c}$ of the coherent state. For the same state considered above $(\alpha$ $=1,\langle x\rangle=\langle p\rangle=0)$, the maximum $F$ is obtained for $\langle x\rangle_{c}=\langle p\rangle_{c}$ $=0$, leading to

$$
\max _{c} \sqrt{F}=\sqrt{\frac{2 \sqrt{\pi}}{\gamma}} \exp \left(\frac{1}{2 \gamma^{2}}\right) \operatorname{erfc}\left(\frac{1}{\sqrt{2} \gamma}\right),
$$

where erfc is the complementary error function. In Fig. 2 we have represented $d_{B}$ as a function of $\gamma$, showing that the minimum distance to coherent states (maximum fidelity) occurs for $\gamma=1.08$ with $\max _{c} F \simeq 0.97$.

\section{SQUEEZING}

Squeezed states are those with fluctuations in $x$ or $p$, or in general of any rotated operator $x_{\theta}=x \cos \theta+p \sin \theta$ for arbitrary $\theta$, below the level established by the coherent states. Different uncertainty measures can be used to establish such a limit.

If we use the variance, for the exponential states with arbitrary $\alpha$ we have $\langle x p+p x\rangle=2\langle x\rangle\langle p\rangle$ so that

$$
\left(\Delta x_{\theta}\right)^{2}=\cos ^{2} \theta(\Delta x)^{2}+\sin ^{2} \theta(\Delta p)^{2} .
$$

For $\alpha=1$ the squeezing condition becomes

$$
\left(\Delta x_{\theta}\right)^{2}=\frac{\gamma^{2}}{2} \cos ^{2} \theta+\frac{1}{\gamma^{2}} \sin ^{2} \theta<\frac{1}{2},
$$

which is satisfied either when $\gamma<1$ or when $\gamma>\sqrt{2}$. Maximum squeezing occurs always for $\theta=0, \pi / 2$, i.e., the squeez- 
ing condition is equivalent to $\Delta_{e} x<\Delta_{c} x$ or $\Delta_{e} p<\Delta_{c} p$.

The result is slightly different if we use the threshold (3.9) established by Rényi entropy so that $\delta_{e} x<\delta_{c} x$ occurs when $\gamma<\sqrt{\pi / 2}$ while $\delta_{e} p<\delta_{c} p$ holds when $\gamma>(4 / 5) \sqrt{\pi / 2}$. Thus all states $\alpha=1$ are squeezed. Moreover, with the Rényi measure it is possible that both $x$ and $p$ are squeezed simultaneously, which holds for $\gamma \in(4 / 5,1) \sqrt{\pi / 2}$.

\section{NUMBER STATISTICS}

A relevant signature of nonclassical behavior (especially in quantum optics) is sub-Poissonian number statistics, which means that the fluctuations of the number operator are below the level established by coherent states.

The number operator is

$$
\hat{n}=\frac{1}{2}\left(x^{2}+p^{2}-1\right),
$$

and the number states $|n\rangle$ are the eigenstates of $\hat{n}, \hat{n}|n\rangle$ $=n|n\rangle, n=0,1, \ldots, \infty$, with wave function

$$
\psi_{n}(x)=\sqrt{\frac{1}{\sqrt{\pi} 2^{n} n !}} H_{n}(x) \exp \left(-x^{2} / 2\right),
$$

where $H_{n}$ are the Hermite polynomials. The number statistics for an arbitrary state $|\psi\rangle$ is

$$
\mathcal{P}(n)=|\langle n \mid \psi\rangle|^{2} \text {. }
$$

Concerning exponential states, the variance is of little use since from Eq. (3.11) we have $\left\langle p^{4}\right\rangle \rightarrow \infty$ so that $\Delta_{e} n \rightarrow \infty$. For a more accurate assessment of number fluctuations $\delta n$ we can rely on the Rényi entropy

$$
\delta_{j} n=\frac{1}{\sum_{n=0}^{\infty} \mathcal{P}_{j}^{2}(n)},
$$

for $j=e, c$, so we may say that the analog of sub-Poissonian statistics for this measure is $\delta_{e} n<\delta_{c} n$ provided that the two states have the same mean number $\langle\hat{n}\rangle_{e}=\langle\hat{n}\rangle_{c}$. For the exponential states with $\alpha=1$ and $\langle x\rangle=\langle p\rangle=0$ we have that $\langle\hat{n}\rangle_{e}$ $=\langle\hat{n}\rangle_{c}$ is just a function of $\gamma$. In Fig. 3 we have represented both $\delta_{e} n, \delta_{c} n$ for $\alpha=1$ as functions of $\gamma$, showing that the analog of sub-Poissonian behavior (i.e., $\delta_{e} n<\delta_{c} n$ ) occurs for all $\gamma$. We can note that this is fully opposite to the predictions of variance.

\section{HEISENBERG LIMIT}

Quantum fluctuations establish a limit to the resolution of precision measurements, which is usually expressed as a function of the energy resources employed in the measurement. This is the case of interferometric measurements devised to detect small phase shifts $\phi$ generated by the number operator $\hat{n}$, which are rotations in the $x, p$ plane. Phase shifts are detected provided that $\phi$ is above a threshold $\Delta \phi$ imposed by quantum fluctuations. In most cases, this threshold can be determined simply by a propagation of uncertainties

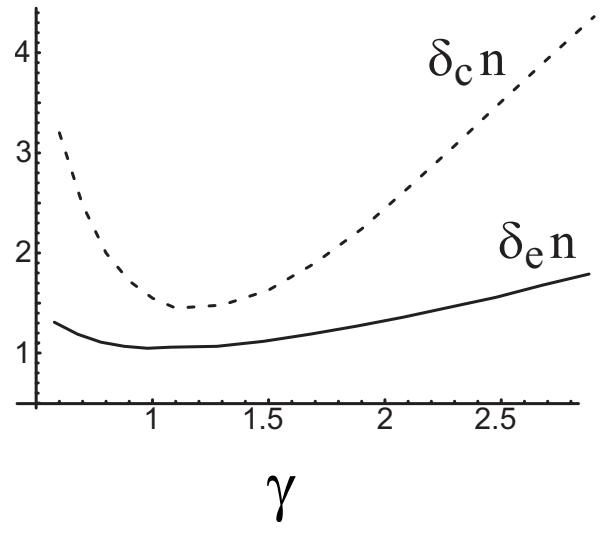

FIG. 3. Number uncertainty $\delta_{e} n$ (solid line) as a function of $\gamma$ for exponential states with $\alpha=1$ and $\langle x\rangle=\langle p\rangle=0$, compared with the number uncertainty for a coherent state $\delta_{c} n$ (dashed line) with the same mean number $\langle\hat{n}\rangle_{e}=\langle\hat{n}\rangle_{c}$.

from the measured observable $M$ to the inferred value of the phase shift $\phi(M)$ so that, using the variance for example [16],

$$
\Delta \phi=\left|\frac{\partial \phi}{\partial\langle M\rangle}\right| \Delta M=\frac{\Delta M}{|\partial\langle M\rangle / \partial \phi|} .
$$

For definiteness, let us consider $M=x$ so that [16]

$$
\left|\frac{\partial\langle x\rangle}{\partial \phi}\right|=|\langle[x, \hat{n}]\rangle|=|\langle p\rangle|
$$

and

$$
\Delta \phi=\frac{\Delta x}{|\langle p\rangle|} .
$$

In order to optimize $\Delta \phi$ let us consider that the state experiencing the phase shift satisfies $\langle x\rangle=0$ so that $|\langle p\rangle|$ is maximum for fixed energy resources, represented in this case by the mean number $\langle\hat{n}\rangle$. Furthermore, we shall consider $\Delta x$ $\rightarrow 0$. This is illustrated in Fig. 4 .

Since $\Delta x \rightarrow 0$ implies $\Delta p \gg \Delta x$, we can use the approximation

$$
\langle\hat{n}\rangle \simeq \frac{1}{2}\left[(\Delta p)^{2}+\langle p\rangle^{2}\right],
$$

so that

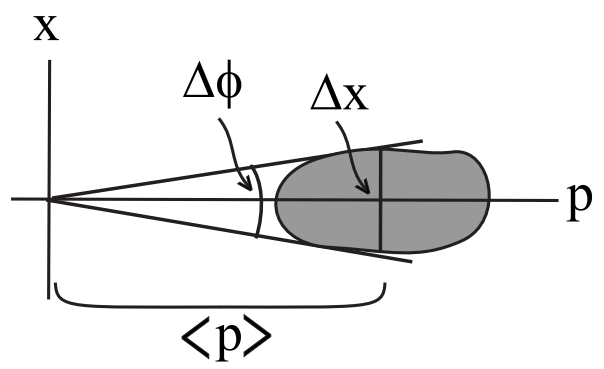

FIG. 4. Phase uncertainty in Eq. (7.3) where the shadowed region represents the area of uncertainty. 


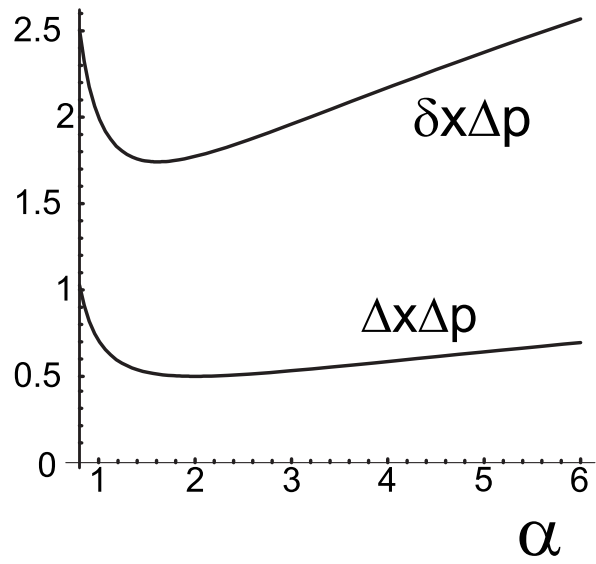

FIG. 5. $\Delta x \Delta p$ and $\delta x \Delta p$ for the states (3.1) as functions of $\alpha$.

$$
\Delta \phi=\frac{\Delta x}{\sqrt{2\langle\hat{n}\rangle-(\Delta p)^{2}}} .
$$

Focusing on exponential states and minimizing with respect to $\gamma$, we get

$$
\Delta \phi=\frac{\Delta x \Delta p}{\langle\hat{n}\rangle},
$$

which is reached for

$$
\gamma=\frac{\alpha 2^{1 / \alpha}}{2 \sqrt{\langle\hat{n}\rangle}} \sqrt{\frac{\Gamma(2-1 / \alpha)}{\Gamma(1 / \alpha)}}
$$

with

$$
\Delta x \Delta p=\frac{\alpha \sqrt{\Gamma(3 / \alpha) \Gamma(2-1 / \alpha)}}{2 \Gamma(1 / \alpha)},
$$

which does not depend on $\langle\hat{n}\rangle$.

Similarly, using Rényi entropy instead of variance to assess the uncertainty of $M$ we arrive at a closely related result

$$
\delta \phi=\frac{\delta x \Delta p}{\langle\hat{n}\rangle},
$$

with

$$
\delta x \Delta p=2^{1 / \alpha} \sqrt{\Gamma(1 / \alpha) \Gamma(2-1 / \alpha)},
$$

for the same $\gamma$ as above. There is a lack of symmetry between $x$ and $p$ in Eqs. (7.9) and (7.10). This mixture is unavoidable since $\delta x$ arises from using the Rényi entropy to assess the uncertainty of the measured observable, while $\Delta p$ unavoidably arises when expressing $\langle p\rangle$ in terms of the mean value of the number operator in Eq. (7.4), since $\left\langle p^{2}\right\rangle$ $=(\Delta p)^{2}+\langle p\rangle^{2}$ irrespective of the uncertainty measure adopted for $x$.

We get the result that all exponential states reach the Heisenberg limit, which is the best accuracy allowed by the quantum theory for the detection of linear phase shifts generated by $\hat{n}$. This is because the minimum phase uncertainty scales as the inverse of the mean number [16]. In Fig. 5 we have represented both $\Delta x \Delta p$ and $\delta x \Delta p$ for the exponential states as functions of $\alpha$, showing that the optimum states are given by $\alpha \simeq 2$ (i.e., Gaussians) or $\alpha \simeq 1.6$, respectively.

\section{CONCLUSIONS}

We have examined the quantum properties of exponential states. These states present a lesser position-momentum uncertainty product than the minimum uncertainty states of variance, they are nonclassical states with slightly negative Wigner function, present squeezing, satisfy the analog of sub-Poissonian number statistics, and allow us to reach the Heisenberg limit. These states are feasible in practice since they are the bound states of $\delta$ potentials, which can be suitable approached via square wells.

Quantum fluctuations are involved in fundamental concepts such as quantum versus classical behavior, complementarity, and quantum limits to precision measurements. There is the peculiarity that the quantum theory does not impose nor does it suggest any particular uncertainty measure, while the results obtained in these areas depend on the choice adopted. This is the case, for example, of complementarity [17]. Most of the works on quantum fluctuations deal exclusively with variance. However, there is nothing in the quantum formalism favoring variance, and, as we have mentioned above, it can encounter relevant disadvantages. Thus, alternative approaches such as the one considered in this paper are worth elaborating.

\section{ACKNOWLEDGMENT}

A.L. acknowledges support from Project No. PR1-A/0715378 of the Universidad Complutense.

\section{APPENDIX: UNCERTAINTY RELATIONS WITH RÉNYI ENTROPIES}

Here we provide a simple derivation of the uncertainty relations presented in Ref. [6]. The state of the system in a Hilbert space of dimension $N$ is represented by a density matrix $\rho$, while $\rho_{a, b}$ will represent the reduced density matrices

$$
\rho_{a}=\sum_{a} \mathcal{P}(a)|a\rangle\left\langle a\left|, \quad \rho_{b}=\sum_{b} \mathcal{P}(b)\right| b\right\rangle\langle b|,
$$

where $\mathcal{P}(j)=\langle j|\rho| j\rangle, j=a, b$, are the statistics of observables represented by two Hermitian operators $A, B,|a\rangle$ and $|b\rangle$ being the corresponding eigenvectors. We consider complementary observables so that $|\langle a \mid b\rangle|=1 / \sqrt{N}$.

Following the approach in Ref. [6], the derivation of uncertainty relations is simplified if we regard the above density matrices as vectors,

$$
\mathbf{x}=\tilde{\rho_{a}}, \quad \mathbf{y}=\tilde{\rho_{b}}, \quad \mathbf{v}=\tilde{\rho},
$$

where the tilde above any operator $W$ designates

$$
\widetilde{W}=W-\frac{1}{N} I,
$$

and $I$ is the $N \times N$ identity. A scalar product between arbitrary vectors $\mathbf{v}_{F, G}$ is defined via the trace of the product of the corresponding operators $F, G$ in the form 


$$
\mathbf{v}_{F} \cdot \mathbf{v}_{G}=\operatorname{tr}\left(F^{\dagger} G\right) .
$$

It can be seen that complementarity implies that $\mathbf{x} \cdot \mathbf{y}=0$, and it follows that

$$
\begin{gathered}
\mathbf{x}^{2}=\mathbf{v} \cdot \mathbf{x}=\mathcal{C}_{A}^{2}-\frac{1}{N}, \\
\mathbf{y}^{2}=\mathbf{v} \cdot \mathbf{y}=\mathcal{C}_{B}^{2}-\frac{1}{N}, \\
\mathbf{v}^{2}=\operatorname{tr}\left(\rho^{2}\right)-\frac{1}{N},
\end{gathered}
$$

where

$$
\mathcal{C}_{A}^{2}=\sum_{a} \mathcal{P}^{2}(a)=\frac{1}{\delta A},
$$

and similarly for $\mathcal{C}_{B}$.

Considering an orthogonal decomposition of $\mathbf{v}$ of the form

$$
\mathbf{v}=\alpha_{x} \mathbf{x}+\alpha_{y} \mathbf{y}+\alpha_{z} \mathbf{z}
$$

where $\alpha_{x, y, z}$ are scalars and $\mathbf{z} \perp \mathbf{x}, \mathbf{y}$, we get from Eq. (A5) that $\alpha_{x}=\alpha_{y}=1$ so that

$$
\mathbf{x}^{2}+\mathbf{y}^{2} \leqslant \mathbf{v}^{2}
$$

which is equivalent to

$$
\mathcal{C}_{A}^{2}+\mathcal{C}_{B}^{2} \leqslant \frac{N+1}{N}, \quad \mathcal{C}_{A}^{2} \mathcal{C}_{B}^{2} \leqslant\left(\frac{N+1}{2 N}\right)^{2}
$$

The last one can be expressed also as

$$
\delta A \delta B \geqslant\left(\frac{2 N}{N+1}\right)^{2} .
$$

A weaker relation has been obtained via a slightly different method in Ref. [7],

$$
\delta A \delta B \geqslant\left(\frac{2 \sqrt{N}}{\sqrt{N}+1}\right)^{2} .
$$

[1] D. M. Meekhof, C. Monroe, B. E. King, W. M. Itano, and D. J. Wineland, Phys. Rev. Lett. 76, 1796 (1996).

[2] J. Peřina, Quantum Statistics of Linear and Nonlinear Optical Phenomea, 2nd ed. (Kluwer Academic, Dordrecht, 1991); L. Mandel and E. Wolf, Optical Coherence and Quantum Optics (Cambridge University Press, Cambridge, U.K., 1995).

[3] J. Hilgevoord, Am. J. Phys. 70, 983 (2002); G. N. Lawrence, Laser Focus World 30, 109 (1994); J. Řeháček and Z. Hradil, J. Mod. Opt. 51, 979 (2004).

[4] A. Wehrl, Rep. Math. Phys. 30, 119 (1991); B. Mirbach and H. J. Korsch, Ann. Phys. (N.Y.) 265, 80 (1998); S. Gnutzmann and K. Życzkowski, J. Phys. A 34, 10123 (2001); V. Majerník and E. Majerníková, Rep. Math. Phys. 47, 381 (2001).

[5] E. J. Heller, Phys. Rev. A 35, 1360 (1987); H. Maassen and J. B. M. Uffink, Phys. Rev. Lett. 60, 1103 (1988); I. BialynickiBirula, M. Freyberger, and W. Schleich, Phys. Scr., T 48, 113 (1993); Č Brukner and A. Zeilinger, Phys. Rev. Lett. 83, 3354 (1999); Phys. Rev. A 63, 022113 (2001); M. J. W. Hall, ibid. 59, 2602 (1999); A. Luis, ibid. 66, 013806 (2002); J. Phys. A 35, 8805 (2002); I. Bialynicki-Birula, Phys. Rev. A 74, 052101 (2006).

[6] U. Larsen, J. Phys. A 23, 1041 (1990).

[7] A. Luis, Phys. Rev. A 67, 032108 (2003).
[8] A. Luis, Opt. Lett. 31, 3644 (2006).

[9] S. Gasiorowicz, Quantum Physics, 3rd ed. (John Wiley, New York, 2003).

[10] A. Luis, Phys. Rev. A 64, 012103 (2001).

[11] C. T. Lee, Phys. Rev. A 44, R2775 (1991); 52, 3374 (1995); N. Lütkenhaus and S. M. Barnett, ibid. 51, 3340 (1995); A. F. de Lima and B. Baseia, ibid. 54, 4589 (1996); J. Janszky, M. G. Kim, and M. S. Kim, ibid. 53, 502 (1996).

[12] M. G. Benedict and A. Czirják, Phys. Rev. A 60, 4034 (1999); A. Kenfack and K. Zyczkowski, J. Opt. B: Quantum Semiclassical Opt. 6, 396 (2004).

[13] R. L. Hudson, Rep. Math. Phys. 6, 249 (1974).

[14] A. Luis, Opt. Commun. 266, 426 (2006).

[15] M. Hillery, Phys. Rev. A 35, 725 (1987); P. Marian, T. A. Marian, and H. Scutaru, Phys. Rev. Lett. 88, 153601 (2002); Phys. Rev. A 69, 022104 (2004).

[16] M. Hillery and L. Mlodinow, Phys. Rev. A 48, 1548 (1993); C. Brif and A. Mann, ibid. 54, 4505 (1996); Z. Y. Ou, ibid. 55, 2598 (1997); A. Luis and L. L. Sánchez-Soto, in Progress in Optics, edited by E. Wolf (Elsevier, Amsterdam, 2000), Vol. 41, p. 421.

[17] A. Luis, Phys. Rev. Lett. 88, 230401 (2002). 